\title{
CORRIGENDUM
}

\section{Hypoxia-stimulated cardiac fibroblast production of IL-6 promotes myocardial fibrosis via the TGF- $\beta 1$ signaling pathway}

Jia-Hong Wang, Lan Zhao, Xin Pan, Nan-Nan Chen, Jian Chen, Qun-Lin Gong, Feng Su, Jian Yan, Yan Zhang and Shao-Heng Zhang

Laboratory Investigation (2016) 96, 1035; doi:10.1038/labinvest.2016.84

Correction to: Laboratory Investigation advance online publication 27 June 2016; doi:10.1038/labinvest.2016.65
In the Acknowledgment section of this article, the correct grant number for the Shanghai Health Commission Scientific Research Found Projects is 201440343 (201440343, to WJ-H). 